\title{
Specific Electrocardiograph Intervals Predict Hospitalization with Atrial Fibrillation in Those with Chronic Kidney Disease
}

\author{
Katherine Mikovna Scovner ${ }^{a}$ b Simon Correa ${ }^{a, b}$ \\ Brian L. Claggett ${ }^{\text {b, c }}$ Conor D. Barrett ${ }^{\text {b, d }}$ Sushrut S. Waikar \\ Scott D. Solomon ${ }^{\mathrm{b}, \mathrm{c}}$ Finnian R. Mc Causland ${ }^{\mathrm{a}} \mathrm{b}$ \\ aDivision of Renal Medicine, Brigham and Women's Hospital, Boston, MA, USA; bHarvard Medical School, Boston, \\ MA, USA; 'Department of Cardiovascular Medicine, Brigham and Women's Hospital, Boston, MA, USA; ${ }^{\mathrm{d} D i v i s i o n}$ of \\ Cardiology, Massachusetts General Hospital, Boston, MA, USA; 'Section of Nephrology, Boston University School of \\ Medicine and Boston Medical Center, Boston, MA, USA
}

\section{Keywords}

Atrial fibrillation · Chronic kidney disease $\cdot$ Hospitalization · Arrhythmia

\begin{abstract}
Introduction: Atrial fibrillation (AF) is common in patients with chronic kidney disease (CKD) and is associated with higher rates of hospitalization compared to those without AF. Whether routine electrocardiographic parameters are predictive of future hospitalizations with AF is not clear. Methods: The present study is an analysis of a prospective cohort of 2,759 patients without baseline AF from the Chronic Renal Insufficiency Cohort, a large prospective multicenter study of patients with nondialysis-dependent CKD. Unadjusted and adjusted Cox regression models were fit to examine the association of baseline categories of QTc, QRS, and PR intervals with time to first hospitalization with AF. Restricted cubic splines were used to display nonlinear associations. Results: The mean age of subjects at baseline was $58 \pm 11$ years, $55 \%$ were male, and $44 \%$ were Black. The mean follow-up was 6.6 years during which 224 participants experienced a hospitalization with AF. The association of baseline QTc interval with risk of AF hospitalization was nonlinear, such that the lowest and highest quartiles of QTc $(<407$ and
\end{abstract}

$>431 \mathrm{~ms}$, respectively) had higher adjusted risk of AF hospitalization, compared with the second quartile (407-416 ms) (aHR Q1:Q2 1.58, 95\% Cl 1.03-2.41; $p=0.03$; aHR Q4:Q2 1.84, $95 \% \mathrm{Cl} 1.22-2.78 ; p<0.01)$. Longer QRS was associated with a higher risk of hospitalization with AF among the subgroup of patients with a history of heart failure (HF). PR interval was not associated with AF hospitalization. Discussion/Conclusion: The association of QTc with risk for hospitalization with AF among patients with CKD is nonlinear, while the association of longer QRS with AF hospitalization is restricted to patients with baseline HF. Electrocardiography may represent a simple and widely accessible method for risk stratification of future $A F$ in patients with CKD.

(c) 2021 S. Karger AG, Basel

\section{Introduction}

Chronic kidney disease (CKD) affects 37 million Americans ( $>1$ in 7 adults) [1] and is the 9th leading cause of death in the United States [2]. CKD patients over the age of 65 years are twice as likely to have cardiovascular disease compared with the general population $(64.5 \%$ compared to 32.4\%) [3]. As CKD patients are more likely to die from cardiovascular causes than to develop end- 
stage renal disease $[4,5]$, early identification of risk factors for cardiovascular complications is particularly important in this patient population.

Atrial fibrillation (AF) is the most common arrhythmia in patients with CKD, with a prevalence of $24 \%$ in those over the age of 65 , compared to $10 \%$ in those without CKD [3]. A longer QT interval, reflecting the duration of ventricular depolarization and repolarization, is associated with a higher incidence of AF in American adults [6]. Early-onset AF, defined as occurring in patients below the age of 50 years, has also been demonstrated to be more prevalent in individuals with genetic long QT syndromes, compared with the general population ( 2 vs. $0.1 \%$, respectively) $[7,8]$. Prolongation of the QRS interval, which corresponds to ventricular depolarization, is associated with ventricular structural and functional abnormalities $[9,10]$ and has also been associated with a higher AF prevalence [10]. The association of the PR interval (the time it takes for an electrical impulse to move from the sinus node through the atrioventricular node to the Purkinje fibers) with incident AF is less clear, with some studies suggesting a higher risk of AF with PR prolongation [11-13], while others do not [14].

As AF is present in approximately one quarter of the CKD population over the age of 65 [3] and is associated with adverse cardiovascular outcomes [15], identification of simple predictors of hospitalization with AF in CKD patients may optimize risk stratification in this vulnerable population. In the present study, we use data from the Chronic Renal Insufficiency Cohort (CRIC) study to determine the association of QTc, QRS, and PR intervals with AF hospitalizations.

\section{Materials and Methods}

\section{Study Design and Population}

The CRIC is a prospective, multicenter study funded by the National Institute of Diabetes and Digestive and Kidney Diseases. The methods of this study have been previously described $[16,17]$. In brief, the study enrolled 3,939 subjects aged 21-74 from June 2003 to September 2008 with mild to moderate CKD (estimated glomerular filtration rate [eGFR] $20-70 \mathrm{~mL} / \mathrm{min}$ per $1.73 \mathrm{~m}^{2}$ ), half of whom had diabetes mellitus (DM) and half of whom were female. Subjects had electrocardiography (ECG) obtained at their baseline in-center visits. Exclusion criteria included institutionalization (e.g. imprisonment and nursing home residency), inability to provide consent, inability to participate in study procedures, New York Heart Association class III or IV heart failure (HF) at baseline, cirrhosis, HIV and/or AIDS, pregnancy, prior history of dialysis, prior organ or bone marrow transplant, immunosuppressive therapy for primary renal disease or vasculitis within the preceding 6 months, previous chemotherapy or alkylating agents for systemic cancer other than nonmelanoma skin cancer in the preceding 2 years, polycystic kidney disease, or participation in another research study. For the purposes of this study, we restricted our analysis to the of 2,759 patients who did not have baseline AF. Follow-up continued until death, withdrawal of consent, loss to follow-up, or through March 2013. Written informed consent was provided by all participants. The institutional review boards at all participating centers approved and oversaw the CRIC study procedures.

\section{Exposure}

The primary exposure in the present study is the QTc interval obtained from a 12-lead ECG at the baseline visit. Secondary exposures include the QRS and PR intervals from the same visit. These exposures were initially assessed as quartiles to allow for the possibility of nonlinear associations. All baseline 12-lead ECGs were performed using a standardized protocol with a GE MAC 1200 electrocardiograph (GE Healthcare, Wauwatosa, WI, USA). ECGs were transmitted to the CRIC ECG Reading Center at the Wake Forest School of Medicine, Winston-Salem, North Carolina, for interpretation.

\section{Study Outcomes}

The primary outcome of interest was hospitalization with AF, which was a prespecified end point of the CRIC study. Patients were asked whether they had been to the emergency department or admitted as part of their biannual query, which alternated as occurring at in-person study visits or via phone call visits. If a patient had presented to the hospital, hospital records were assessed for the International Classification of Diseases, Ninth Revision code 427.31 for AF [18]. If a code for AF was found in the medical records, clinical data and ECGs were sent to a centralized location for manual review by at least 2 physicians prior to final adjudication for the presence of AF.

\section{Statistical Analysis}

Continuous variables were described as means \pm standard deviation; categorical variables were described as proportions (percentages). Baseline characteristics, according to baseline ECG quartiles, were compared with tests for trend based on linear regression, $\chi^{2}$ trend test, and the Cuzick nonparametric trend test [19]. Unadjusted and subsequently adjusted Cox proportional hazards models were fit to evaluate the association of quartiles of QTc, QRS, and PR intervals with time to hospitalization with AF. Model 1 adjusted for age, sex, and race/ethnicity. Model 2 (main model) was fit to account for the parameters of model 1 and additionally for body mass index (BMI), DM, systolic blood pressure, coronary artery disease (CAD), HF, stroke, eGFR (based on the CKD Epidemiology Collaboration equation) [20], log-transformed 24-h urine protein, serum hematocrit, albumin, potassium, and calcium. An exploratory model (model 3) was fit to account for the parameters of model 1 and 2 and additionally for the baseline use of angiotensin-converting enzyme inhibitors (ACEI) or angiotensin receptor blockers (ARB), $\beta$-blockers, potassiumsparing diuretics, loop diuretics, thiazide diuretics, statin medications, and warfarin. The proportional hazard assumption was checked in all models through visualization of Schoenfeld residuals, log-log plots, and global tests for the assumption. We tested for the presence of effect modification according to baseline HF or baseline eGFR via inclusion of cross-product terms in model 2 (the 
Table 1. Baseline characteristics by QTc quartiles

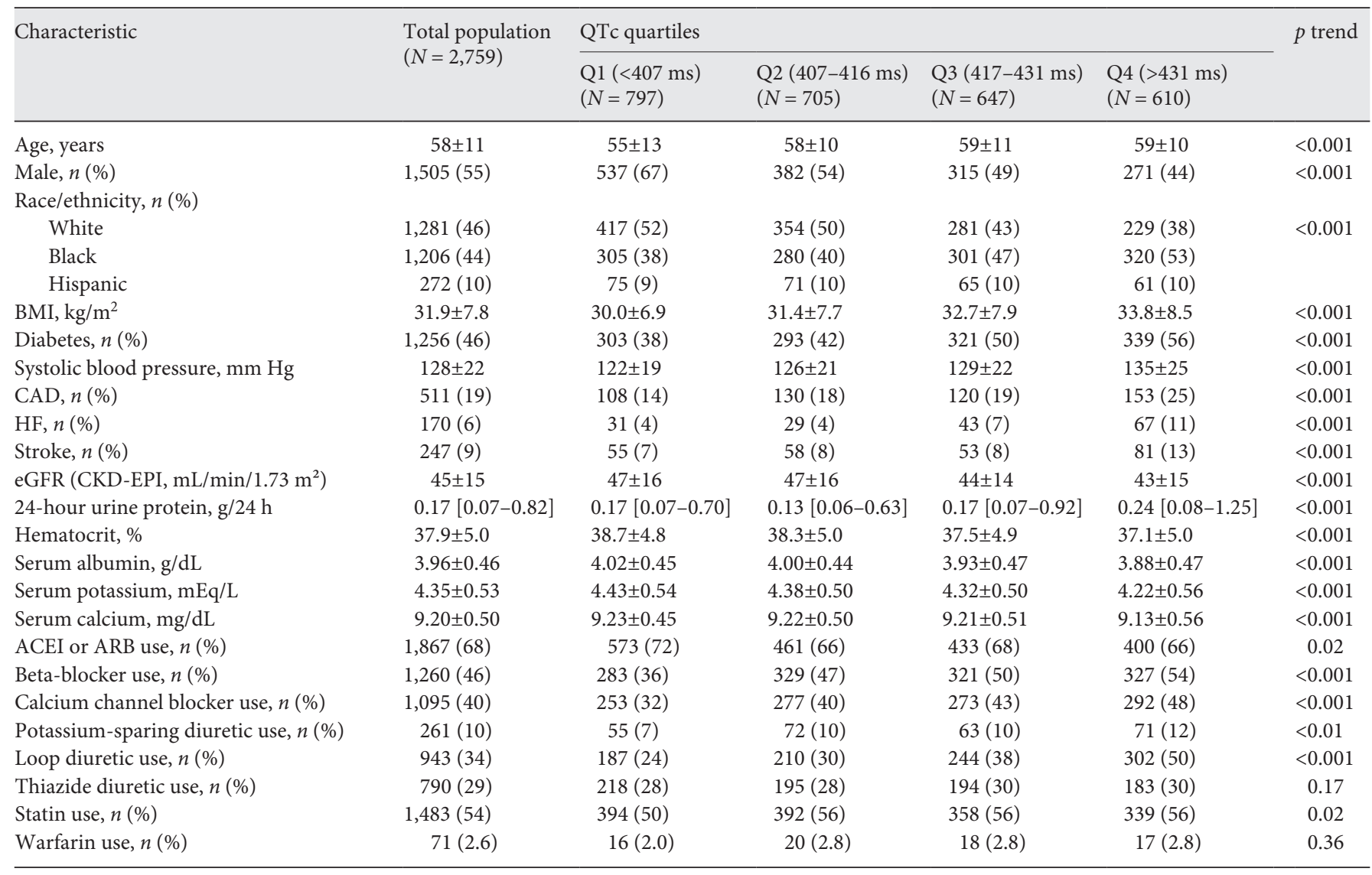

Continuous variables presented as mean \pm standard deviation if normally distributed or median [25th-75th percentile] if nonnormally distributed; categorical variables presented as count (\%). Baseline characteristics, according to quartiles of QTc, were compared with tests for trend based on linear regression, $\chi^{2}$ trend test, and the Cuzick nonparametric trend test. eGFR, estimated glomerular filtration rate; CKD-EPI, Chronic Kidney Disease Epidemiology Collaboration; ACEI, angiotensin-converting enzyme inhibitor; ARB, angiotensin receptor blocker; HF, heart failure; BMI, body mass index; CAD, coronary artery disease.

main adjusted model). The lowest quartile was chosen as the reference for all initial analyses. If there was evidence for nonlinearity, then quartile 2 was chosen as the reference. Flexible cubic spline analyses were performed to further assess for the pattern of nonlinear associations. The spline model fit and number of knots were determined by Akaike's and Bayesian information criteria. A 2 -sided $p$ value $<0.05$ was considered to be statistically significant. Analyses were completed using STATA 14.2 (College Station, TX, USA).

\section{Results}

\section{QTC Intervals and AF Hospitalization}

Baseline Characteristics according to QTc Quartiles

Baseline ECG parameters were measured in 2,759 CRIC study participants who did not have AF at baseline. The mean follow-up for these patients was 6.6 years. A first hospitalization with AF event occurred in 224 of these subjects. The mean baseline QTc interval was $418 \pm$ 23 ms. Subjects with longer QTc were more likely to be older, female, Black, have higher BMI, and to have DM, higher systolic blood pressure, CAD, HF, history of stroke, higher 24-h urine protein, and were more likely be on a $\beta$-blocker, calcium channel blocker, potassium-sparing diuretic, loop diuretic, and a statin. They had lower baseline eGFR, hematocrit, albumin, potassium, and calcium. They were less likely to be prescribed an ACEI or ARB. Baseline characteristics for the study population by QTc quartile are shown in Table 1.

\section{Risk of Hospitalization with AF with Longer QTc Interval: Categorical Models}

The association of quartiles of QTc with AF hospitalization was nonlinear $(p=0.03)$ (shown in Fig. 1); there- 


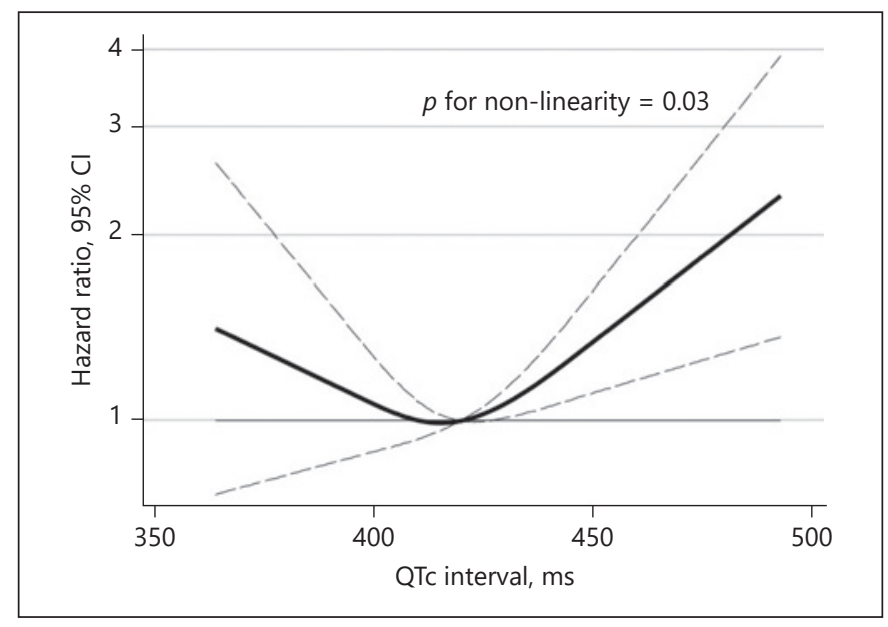

Fig. 1. Estimated risk of hospitalization with AF according to baseline QTc interval in the main adjusted model (association adjusted for age, sex, race/ethnicity, BMI, DM, systolic blood pressure, CAD, HF, stroke, eGFR [via CKD-EPI equation], log-transformed 24-h urine protein, serum hematocrit, serum albumin, serum potassium, serum calcium, and clinical center). Estimated risk relative to reference value of $420 \mathrm{~ms}$. AF, atrial fibrillation; DM, diabetes mellitus; CKD, chronic kidney disease; HF, heart failure; $\mathrm{BMI}$, body mass index; CAD, coronary artery disease; eGFR, estimate glomerular filtration rate; CKD-EPI, Chronic Kidney Disease Epidemiology Collaboration.

fore, quartile 2 was chosen as the reference. In the unadjusted model, the highest quartile was associated with higher risk of hospitalization with $\mathrm{AF}$, compared to the second quartile, while the lowest quartile was not (HR Q4:Q2 2.21, 95\% CI 1.50-3.25; $p<0.001$; HR Q1:Q2 1.27, $95 \%$ CI $0.85-1.89 ; p=0.24)$. However in the adjusted model, both the highest and lowest quartiles of QTc were associated with a higher risk of hospitalization with AF, compared to the second quartile (aHR Q4:Q2 1.84, 95\% CI 1.22-2.78; $p<0.01$; aHR Q1:Q2 1.58, 95\% CI 1.03$2.41 ; p=0.03$ ) (Table 2). These patterns of associations persisted in the exploratory model that further adjusted for medication use (aHR Q4:Q2 1.89, 95\% CI 1.25-2.86; $p<0.01$; aHR Q1:Q2 1.69, 95\% CI 1.10-2.59; $p=0.02$ ). The cumulative incidence of hospitalization with AF by baseline QTc quartile is shown in Figure 2.

Risk of Hospitalization with AF with Longer QTc

Interval: Continuous Models

The evidence for a nonlinear association of QTc with AF hospitalization was demonstrated by the flexible cubic spline model (shown in Fig. 1). Based on this analysis, the lowest risk for hospitalization with AF corresponded to a QTc of approximately $420 \mathrm{~ms}$. In the main adjusted mod- el, each $10 \mathrm{~ms}$ increase in QTc from $420 \mathrm{~ms}$ was associated with a $14 \%$ increased risk of hospitalization with $\mathrm{AF}$ (aHR 1.14, 95\% CI 1.04-1.25; $p<0.01$ ); each decrease in QTc of $10 \mathrm{~ms}$ from $420 \mathrm{~ms}$ was not (aHR 0.88, 95\% CI $0.78-1.01 ; p=0.07)$.

Risk of Hospitalization with AF with Longer QTc Interval: Effect Modification

There was no evidence for effect modification of the association of QTc interval with AF hospitalization according to baseline HF $(P$-interaction $=0.85)$ or quartiles of eGFR $(P$-interaction $=0.74)$ in the main adjusted model.

\section{QRS Interval and AF Hospitalization}

Baseline Characteristics according to QRS Quartiles

The mean QRS interval was $95 \pm 16 \mathrm{~ms}$. Subjects with longer QRS were more likely to be older, male, Caucasian, have higher BMI, have higher systolic blood pressure, $\mathrm{CAD}, \mathrm{HF}$, history of stroke, more proteinuria, higher hematocrit, and had a higher likelihood of being on an ACEI or ARB, $\beta$-blocker, calcium channel blocker, loop diuretic, statin, and warfarin. They had lower eGFR and lower potassium levels. Baseline characteristics for the study population by QRS quartile are shown in online suppl. Table 1; see www.karger.com/doi/10.1159/000515670 for all online suppl. material.

\section{Risk of Hospitalization with AF with Longer QRS \\ Interval: Categorical Models}

In the unadjusted model, QRS in the highest quartile (Q4) compared to the lowest quartile (Q1; reference) was associated with a higher risk of hospitalization with AF (aHR Q4:Q1 2.00, 95\% CI 1.38-2.90; $p<0.001$ ). However in the main adjusted model, QRS in the highest quartile (Q4) compared to the lowest quartile (Q1; reference) was not associated with risk of hospitalization with AF (aHR Q4:Q1 1.21, 95\% CI 0.80-1.83; $p=0.37$ ) (online suppl. Table 2).

\section{Risk of Hospitalization with AF with Longer QRS}

Interval: Continuous Models

There was no evidence of nonlinearity in the association of QRS with hospitalization with AF in our main adjusted flexible cubic spline analysis $(p=0.76)$ (shown in online suppl. Fig. 1a). While there was an association of longer QRS interval with hospitalization with AF in the unadjusted model (for each $10 \mathrm{~ms}$ increase in QRS, HR 1.14, 95\% CI $1.06-1.22 ; p<0.001)$, this association did not persist in the main adjusted model (for each $10 \mathrm{~ms}$ increase in QRS, aHR $1.02,95 \%$ CI $0.94-1.11 ; p=0.62$ ) (online suppl. Table 2). 
Table 2. Risk of AF hospitalization according to QTc quartiles

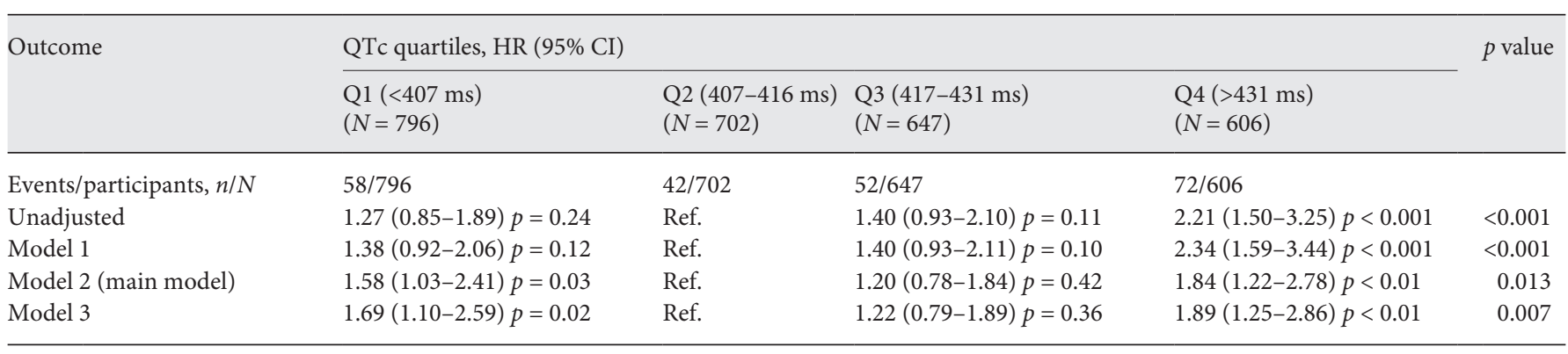

Model 1 adjusted for age, sex, and race/ethnicity. Model 2 adjusted for covariates of model 1 and additionally for baseline body mass index, diabetes mellitus, systolic blood pressure, coronary artery disease, heart failure, stroke, estimate glomerular filtration rate (via Chronic Kidney Disease Epidemiology Collaboration equation), log-transformed 24-h urine protein, serum hematocrit, serum albumin, serum potassium, and serum calcium. Model 3 adjusted for covariates of model 1 and 2 and additionally for baseline use of angiotensin-converting enzyme inhibitor or angiotensin receptor blocker medications, $\beta$-blockers, potassium-sparing diuretics, loop diuretics, thiazide diuretics, statins, and warfarin. AF, atrial fibrillation; HR, hazard ratio; CI, confidence interval.

Risk of Hospitalization with AF with Longer QRS

Interval: Effect Modification

There was no evidence for effect modification according to baseline eGFR $(p=0.18)$ in the main adjusted model. However, there was evidence for effect modification according to history of HF $(P$-interaction $=0.02)$ such that QTc was associated with an increased risk of hospitalization with AF in those with HF in our main adjusted model (for each $10 \mathrm{~ms}$ increase in QRS, aHR 1.36; 95\% CI $1.10-1.69 ; p<0.01$ ), while there was no significant association in those without HF (aHR 0.98; 95\% CI 0.89-1.07; $p=0.62$ ).

\section{$P R$ Interval and AF Hospitalization}

Baseline Characteristics according to PR Quartiles

The mean baseline PR interval was $172 \pm 30 \mathrm{~ms}$. Subjects with longer PR were more likely to be older, male, Black, have higher BMI, to have DM, higher systolic blood pressure, $\mathrm{CAD}, \mathrm{HF}$, history of stroke, to have higher albumin, and were more likely to be on an ACEI or $\mathrm{ARB}, \beta$-blocker, calcium channel blocker, potassiumsparing diuretic, loop diuretic, thiazide diuretic, and statin. They had lower eGFR and lower serum potassium levels. Baseline characteristics for the study population by PR quartile are shown in online suppl. Table 3.

\section{Risk of Hospitalization with AF with Longer PRS \\ Interval: Categorical Models}

In the unadjusted model, PR in the highest quartile (Q4) compared to the lowest quartile (Q1; reference) was associated with an increased risk of hospitalization with AF (HR Q4:Q1 2.22, 95\% CI 1.49-3.30; $p<0.001$ ). How-

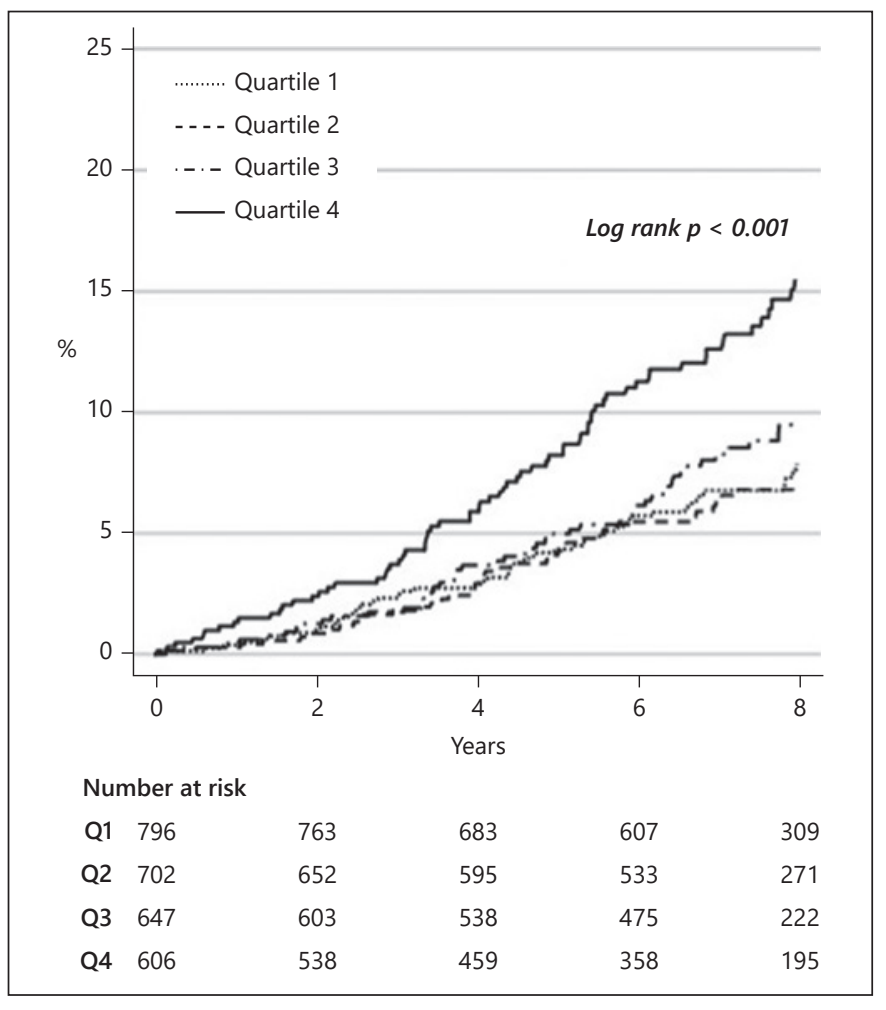

Fig. 2. Cumulative incidence of hospitalization with AF according to baseline QTc quartile. AF, atrial fibrillation.

ever, in the main adjusted model, PR in the highest quartile (Q4) compared to the lowest quartile (Q1; reference) was not associated with risk of hospitalization with $\mathrm{AF}$ (aHR Q4:Q1 1.38, 95\% CI 0.90-2.13; $p=0.14$ ) (online suppl. Table 4). 
Risk of Hospitalization with AF with Longer PR Interval: Continuous Models

There was no evidence of nonlinearity in the association of PR interval with hospitalization with $\mathrm{AF}$ in the main adjusted flexible cubic spline analysis $(p=0.37)$ (shown in online suppl. Fig. 1b). Though there was an association of $\mathrm{PR}$ interval with hospitalization with $\mathrm{AF}$ in the unadjusted model, this did not persist in the main adjusted models (for each $10 \mathrm{~ms}$ increase in PR in the unadjusted model, HR 1.09, 95\% CI 1.05-1.13; $p<0.001$; for each $10 \mathrm{~ms}$ increase in $\mathrm{PR}$ in the adjusted model, aHR 1.03, 95\% CI 0.98-1.07; $p=0.25$ ) (online suppl. Table 4).

\section{Risk of Hospitalization with AF with Longer PR}

Interval: Effect Modification

There was no evidence that the association of the PR interval with AF hospitalization was different according to $\mathrm{HF}(P$-interaction $=0.90)$ or eGFR $(P$-interaction $=$ $0.68)$ in the main adjusted model.

\section{Discussion/Conclusion}

We examined the association of simple ECG measures with the future risk of hospitalization with $\mathrm{AF}$ in patients with CKD participating in the CRIC study. We found that QTc intervals longer than $420 \mathrm{~ms}$ were associated with higher risk for hospitalization with AF, while longer PR intervals were not. The association of longer QRS interval with higher risk of hospitalization with AF was restricted to CRIC participants with HF at baseline.

Several studies have reported a higher prevalence of AF in CKD patients compared with the general population [21]. This is thought to be partially related to common risk factors, such as increased renin-angiotensin-aldosterone system activation, inflammation, heightened sympathetic tone, and myocardial remodeling, which are often associated with both diseases $[21,22]$. As in the general population, AF (vs. none) in patients with CKD is associated with increased risk for stroke and mortality [21]. Indeed, this relationship appears to be bidirectional as the presence of CKD in patients with $\mathrm{AF}$ is associated with all-cause and cardiovascular mortality [23].

As the diagnosis of AF is based on ECG, there has been long-standing interest in identification of other ECG parameters that may predict the future development of AF. A prior analysis of the Atherosclerosis Risk of Communities study reported that longer QTc is associated with an increased risk of incident AF (for each $10 \mathrm{~ms}$ increase in QTc interval, aHR 1.11, 95\% CI 1.07-1.14; $p<0.001$ ) [6].
Furthermore, a higher risk for the development of AF is also seen in individuals with long QT syndromes, compared to those without $[7,8]$. Studies have also demonstrated an association of shorter QRS with less AF. For example, in one cross-sectional study of 25,000 patients with left ventricular dysfunction, QRS $<120 \mathrm{~ms}$ was associated with a lower prevalence of AF compared to QRS $>150 \mathrm{~ms}$ (QRS $<120 \mathrm{~ms}$ compared with $>150 \mathrm{~ms}$, aOR $0.64,95 \%$ CI 0.59-0.69) [10]. With regard to the PR interval, patients with longer PR intervals in the Framingham Heart Study had a higher risk of incident AF (for each $20 \mathrm{~ms}$ increase in PR interval, aHR 1.12, 95\% CI $1.02-1.22 ; p=0.018$ ) [11]. Similarly, having a PR interval greater than or equal to the 95 th percentile was associated with an increased risk of AF in the Copenhagen ECG study (aHR 1.18, 95\% CI 1.06-1.30; $p=0.001$ ) [13], and each standard deviation increase in the PR interval in participants of the Health, Aging, and Body Composition study was also associated with an increased risk of incident AF (aHR 1.13, 95\% CI 1.04-1.23; $p=0.02$ ) [12]. On the other hand, PR interval above $200 \mathrm{~ms}$ was not associated with hospitalization due to AF in a study assessing individuals in the general population (aHR 1.03, 95\% CI $0.74-1.45 ; p=0.85$ ) [14]. Our analyses expand the current knowledge base to the CKD population, which carries an exceptionally high burden of cardiovascular disease.

Several pathophysiologic mechanisms have been suggested to explain the association between long QT and AF in other populations. Central to such hypotheses, prolongation of the QT interval is thought to reflect underlying pathologies that contribute to abnormal ventricular refractoriness, which in turn may predispose to similar abnormalities in atrial refractoriness [6]. This is particularly relevant in the setting of HF, characterized by structural and functional changes in cardiac activity, where QT prolongation and other ECG abnormalities may simply serve as a surrogate marker for other disease processes. For example, atrial enlargement and remodeling (loss of functioning atrial myocardium, regions of low voltage, and spontaneous scarring) in the setting of HF are associated with delayed atrial refractory periods [24-26]. In addition, sinus node remodeling has been observed in the setting of chronic atrial stretch $[27,28]$, and sinus node dysfunction has been implicated in the development of AF [29]. Other studies have found a stronger association between the JT interval (which represents the ventricular repolarization portion of the QT interval) and AF, compared with the QRS portion (which represents ventricular depolarization) [26]. This suggests that abnormalities in ventricular repolarization, rather than depolarization, 
contribute to the increased risk for AF associated with prolonged QT [26]. In our current analyses, the association of QTc increase above $420 \mathrm{~ms}$ with hospitalization with AF persisted even after adjustment for the presence of baseline HF. Notably, we also found that the association of longer QRS with future AF hospitalization was restricted to those with HF at baseline (New York Heart Association class I or II, per CRIC inclusion criteria). Though several explanations have been proposed in the literature, further research is required to elucidate the true role of abnormalities in ventricular repolarization with the development of AF [26].

Our findings are highly relevant, as ECGs are a noninvasive, portable, and widely available tool that may help identify patients at higher risk of developing AF. The relevance is further amplified by the increasing availability of wearable devices, which represent an exciting new forum for rhythm assessment that may identify and predict arrhythmias across patient populations in the future [30]. Furthermore, advances in machine learning are likely to be applied to help predict incident AF in the future [31]. As telemetric technology and machine learning capabilities develop, our ability to use cardiac rhythm information to predict complications will also improve. However, until such techniques are widely available, simple 12-lead ECG data remains affordable and practical to obtain in contemporary care of patients with CKD.

Our study has several strengths. The CRIC study prospectively followed a large number of CKD patients for a prolonged duration (median follow-up time: 6.9 years) with ethnically diverse backgrounds and comorbidities. As with all secondary analyses, however, several limitations exist. First, despite the use of adjusted models, the possibility of residual confounding remains. Furthermore, there is the possibility of ascertainment bias (e.g. patients with longer QTc may have had other comorbidities necessitating closer follow-up and therefore may have been hospitalized more with AF due to closer monitoring). Granular information regarding the initiation of anticoagulation therapy for AF was not available. Further, AF events that were not associated with hospitalization were not captured in this study, though they may still have been complicated by morbidity Repeated measures of ECG parameters would have provided granularity in our data to assess the association between ECG abnormalities and AF outcomes in our patients over time. Lastly, though CKD patients with severe HF may be those who would benefit most from an improved understanding of predictors of $\mathrm{AF}$ hospitalization, patients with NYHA class III and IV HF were excluded from CRIC, limiting the generalizability of our findings to this particularly vulnerable population. Furthermore, whether AF was paroxysmal, persistent, or permanent was not classified in CRIC.

In conclusion, we found that longer QTc and, in those with baseline HF, longer QRS intervals are associated with a higher risk for hospitalization with AF in patients with CKD. Future studies should assess whether serial ECG monitoring for AF development or wearable devices which monitor cardiac rhythms will allow earlier detection and institution of therapy, with the goal of minimizing the morbidity and mortality associated with $\mathrm{AF}$ in patients with CKD.

\section{Acknowledgements}

The original CRIC study was conducted by the CRIC Iinvestigators and supported by the National Institute of Diabetes and Digestive and Kidney Diseases (NIDDK). The data from the CRIC study reported here were supplied by the NIDDK Central Repositories.

\section{Statement of Ethics}

Written informed consent was provided by all participants. The institutional review boards at all participating centers approved and oversaw the CRIC study procedures.

\section{Conflict of Interest Statement}

The authors have no conflicts of interest to declare.

\section{Funding Sources}

Dr. Scovner is supported by the National Institute of Health T32 grant (T32DK 007527). Dr. Solomon is supported by the National Heart, Lung, and Blood Institute grants R01HL141288-01, R01AG060163, and R01HL141505-01A1. Dr. Mc Causland is supported by the National Institute of Diabetes and Digestive and Kidney Diseases grants U01DK096189 and R03DK122240.

\section{Author Contributions}

All co-authors contributed to conception of the study design, data analysis, drafting, and final manuscript writing. All co-authors agree to be accountable for ensuring that all questions related to the accuracy and integrity of any part to the work are appropriately investigated and resolved. 


\section{References}

1 Available from: https://www.cdc.gov/kidneydisease/publications-resources/2019-national-facts.html.

2 Kochanek KD, Murphy SL, Xu J, Arias E. Deaths: final data for 2017. Natl Vital Stat Rep. 2019 Jun;68(9):1-77.

3 United States Renal Data System. End-stage renal disease (ESRD) in the United States CCDiPwCINIoH, National Institute of diabetes and digestive and kidney diseases. In: USRDS annual data report: epidemiology of kidney disease in the United States. Bethesda, MD: National Institutes of Health, National Institute of Diabetes and Digestive and Kidney Health; 2018.

4 Shulman NB, Ford CE, Hall WD, Blaufox MD, Simon D, Langford HG, et al. Prognostic value of serum creatinine and effect of treatment of hypertension on renal function. Results from the hypertension detection and follow-up program. The hypertension detection and Follow-up Program Cooperative Group. Hypertension. 1989 May;13(5 Suppl):I80-93.

5 Sarnak MJ, Levey AS, Schoolwerth AC, Coresh J, Culleton B, Hamm LL, et al. Kidney disease as a risk factor for development of cardiovascular disease: a statement from the American Heart Association Councils on kidney in cardiovascular disease, high blood pressure research, clinical cardiology, and epidemiology and prevention. Circulation. 2003 Nov;108(17):2154-69.

6 Mandyam MC, Soliman EZ, Alonso A, Dewland TA, Heckbert SR, Vittinghoff E, et al. The QT interval and risk of incident atrial fibrillation. Heart Rhythm. 2013 Oct;10(10): $1562-8$.

7 Johnson JN, Tester DJ, Perry J, Salisbury BA, Reed CR, Ackerman MJ. Prevalence of earlyonset atrial fibrillation in congenital long QT syndrome. Heart Rhythm. 2008 May;5(5): 704-9.

8 Zellerhoff S, Pistulli R, Mönnig G, Hinterseer M, Beckmann BM, Köbe J, et al. Atrial arrhythmias in long-QT syndrome under daily life conditions: a nested case control study. J Cardiovasc Electrophysiol. 2009 Apr;20(4): 401-7.

9 Dhingra R, Ho Nam B, Benjamin EJ, Wang TJ, Larson MG, D'Agostino RB, et al. Crosssectional relations of electrocardiographic QRS duration to left ventricular dimensions: the Framingham Heart Study. J Am Coll Cardiol. 2005 Mar 1;45(5):685-9.
10 El-Chami MF, Brancato C, Langberg J, Delurgio $\mathrm{DB}, \mathrm{Bush} \mathrm{H}$, Brosius $\mathrm{L}$, et al. QRS duration is associated with atrial fibrillation in patients with left ventricular dysfunction. Clin Cardiol. 2010 Mar;33(3):132-8.

11 Cheng S, Keyes MJ, Larson MG, McCabe EL, Newton-Cheh C, Levy D, et al. Long-term outcomes in individuals with prolonged $\mathrm{PR}$ interval or first-degree atrioventricular block. JAMA. 2009 Jun 24;301(24):2571-7.

12 Magnani JW, Wang N, Nelson KP, Connelly $S$, Deo R, Rodondi N, et al. Electrocardiographic $\mathrm{PR}$ interval and adverse outcomes in older adults: the health, aging, and Body Composition study. Circ Arrhythm Electrophysiol. 2013 Feb;6(1):84-90.

13 Nielsen JB, Pietersen A, Graff C, Lind B, Struijk JJ, Olesen MS, et al. Risk of atrial fibrillation as a function of the electrocardiographic PR interval: results from the Copenhagen ECG Study. Heart Rhythm. 2013 Sep;10(9):1249-56.

14 Aro AL, Anttonen O, Kerola T, Junttila MJ, Tikkanen JT, Rissanen HA, et al. Prognostic significance of prolonged PR interval in the general population. Eur Heart J. 2014 Jan; 35(2):123-9.

15 Tomaszuk-Kazberuk A, Nikas D, Lopatowska P, Młodawska E, Malyszko J, BachorzewskaGajewska $\mathrm{H}$, et al. Patients with atrial fibrillation and chronic kidney disease more often undergo angioplasty of left main coronary artery: a 867 Patient Study. Kidney Blood Press Res. 2018;43(6):1796-805.

16 Lash JP, Go AS, Appel LJ, He J, Ojo A, Rahman $\mathrm{M}$, et al. Chronic renal insufficiency cohort (CRIC) Study: baseline characteristics and associations with kidney function. Clin J Am Soc Nephrol. 2009 Aug;4(8):1302-11.

17 Snitker S, Doerfler RM, Soliman EZ, Deo R, St Peter WL, Kramlik S, et al. Association of QTprolonging medication use in CKD with electrocardiographic manifestations. Clin J Am Soc Nephrol. 2017 Sep 7;12(9):1409-17.

18 World Health Organization. International classification of diseases: [9th] ninth revision btlwaiI. WHO; 1978.

19 Cuzick J. A Wilcoxon-type test for trend. Stat Med. 1985 Jan-Mar;4(1):87-90.

20 Stevens LA, Coresh J, Schmid CH, Feldman HI, Froissart M, Kusek J, et al. Estimating GFR using serum cystatin $\mathrm{C}$ alone and in combination with serum creatinine: a pooled analysis of 3,418 individuals with CKD. Am J Kidney Dis. 2008 Mar;51(3):395-406.
21 Kulkarni N, Gukathasan N, Sartori S, Baber U. Chronic kidney disease and atrial fibrillation: a contemporary overview. J Atr Fibrillation. 2012 Jun-Jul;5(1):448

22 Hung MJ, Yang NI, Wu IW, Cheng CW, Wu MS, Cherng WJ. Echocardiographic assessment of structural and functional cardiac remodeling in patients with predialysis chronic kidney disease. Echocardiography. $2010 \mathrm{Jul}$ 27(6):621-9.

23 Airy M, Schold JD, Jolly SE, Arrigain S, Bansal $\mathrm{N}$, Winkelmayer WC, et al. Cause-specific mortality in patients with chronic kidney disease and atrial fibrillation. Am J Nephrol. 2018;48(1):36-45.

24 Sanders P, Morton JB, Davidson NC, Spence SJ, Vohra JK, Sparks PB, et al. Electrical remodeling of the atria in congestive heart failure: electrophysiological and electroanatomic mapping in humans. Circulation. 2003 Sep 23;108(12):1461-8

25 Rautaharju PM, Prineas RJ, Wood J, Zhang ZM, Crow R, Heiss G. Electrocardiographic predictors of new-onset heart failure in men and in women free of coronary heart disease (from the Atherosclerosis in Communities [ARIC] Study). Am J Cardiol. 2007 Nov 1; 100(9):1437-41.

26 Patel N, O'Neal WT, Whalen SP, Soliman EZ. The association of QT interval components with atrial fibrillation. Ann Noninvasive Electrocardiol. 2018 Mar;23(2):e12467.

27 Sparks PB, Mond HG, Vohra JK, Jayaprakash S, Kalman JM. Electrical remodeling of the atria following loss of atrioventricular synchrony: a long-term study in humans. Circulation. 1999 Nov 2;100(18):1894-900.

28 Morton JB, Sanders P, Vohra JK, Sparks PB, Morgan JG, Spence SJ, et al. Effect of chronic right atrial stretch on atrial electrical remodeling in patients with an atrial septal defect. Circulation. 2003 Apr 8;107(13):1775-82.

29 Kezerashvili A, Krumerman AK, Fisher JD. Sinus node dysfunction in atrial fibrillation: cause or effect? J Atr Fibrillation. 2008 SepNov; 1(3):30.

30 Perez MV, Mahaffey KW, Hedlin H, Rumsfeld JS, Garcia A, Ferris T, et al. Large-scale assessment of a smartwatch to identify atrial fibrillation. N Engl J Med. 2019 Nov 14; 381(20):1909-17.

31 Hill NR, Ayoubkhani D, McEwan P, Sugrue DM, Farooqui U, Lister S, et al. Predicting atrial fibrillation in primary care using machine learning. PLoS One. 2019;14(11): e0224582. 